\title{
Preparation and Characterization of Bamboo-based
}

\section{Activated Carbons as Electrode Materials for Electric}

\section{Double Layer Capacitors}

Yong-Jung Kim ${ }^{\mathrm{a},{ }^{*}}$, Byoung-Ju Lee ${ }^{\mathrm{b}}$, Hiroaki Suezaki ${ }^{\mathrm{b}}$, Teruaki Chino ${ }^{\mathrm{b}}$, Yusuke Abe ${ }^{\mathrm{b}}$, Takashi Yanagiura ${ }^{\mathrm{b}}$, Ki Chul Park ${ }^{\mathrm{b}}$, Morinobu Endo ${ }^{\mathrm{a}, \mathrm{b}}$

a Institute of Carbon Science \& Technology, Shinshu University, 4-17-1 Wakasato, Nagano 380-8553, Japan

${ }^{\mathrm{b}}$ Department of Electric and Electronic Engineering, Faculty of Engineering, Shinshu University, 4-17-1 Wakasato, Nagano 380-8553, Japan

${ }^{*}$ Corresponding author. Tel: +81-26-269-5655; Fax: +81-26-269-5208

E-mail address: yjk@endomoribu.shinshu-u.ac.jp (Y. J. Kim) 
The everyday use of disposable bamboo-based products has prompted us to recycle their waste into carbon materials for adsorbents [1, 2] and electrodes for electric double layer capacitors (EDLCs). Hitherto diverse carbon precursors including abundant biomass materials have been investigated to prepare activated carbons (ACs) as electrode materials with high surface area, large porosity and homogeneous pore size [3-5]. In general, the pore structure and size of ACs strongly depend on the types of their precursor and activation process [6]. In this work, the activation of bamboo-based carbons was examined at various ratios of $\mathrm{KOH}$. Furthermore, the specific capacitance of the bamboo-based AC electrodes was evaluated using two different kinds of (aqueous and nonaqueous) electrolytes with different ion sizes, whereby detailed information about the structure and size of the developed pores has been obtained.

As a typical procedure, a pristine bamboo was fragmented into pieces (without dehydration), and heated at $700{ }^{\circ} \mathrm{C}$ for $2 \mathrm{~h}$ in an argon (Ar) flow (500 ml/min). After drying at $120^{\circ} \mathrm{C}$ overnight, the bamboo char mixed with $\mathrm{KOH}$ was heated at $800{ }^{\circ} \mathrm{C}$ for $1 \mathrm{~h}$ in an Ar flow $(800 \mathrm{ml} / \mathrm{min})$. The heating rate was $10^{\circ} \mathrm{C} / \mathrm{min}$ in all experiments. The resulting ACs was washed well with deionized water, and dried in vacuo at $80{ }^{\circ} \mathrm{C}$ for 24 h. According to the mixing ratios (bamboo-based char/KOH), the samples were designated as OBC (no addition of KOH), OBK1 (1/1), OBK2 (1/2), OBK3 (1/3) and OBK4 (1/4). The details of the capacitance measurement have been described in the previous report [7].

The fundamental properties of the bamboo-based char and ACs are summarized in Table 1. The relative mesopore fraction volume $\left(\mathrm{V}_{\text {meso }} / \mathrm{V}_{\text {micro }}\right)$ and area $\left(\mathrm{S}_{\text {meso }} / \mathrm{S}_{\text {micro }}\right)$ exhibited the highest values in the OBK3 sample. In addition, the apparent density ( $\rho$ ) of the OBK3 sample was the lowest due to the high percentage of the large pore volume 
$\left(\mathrm{V}_{\text {tot }}-\mathrm{V}_{\text {micro }}\right)$. The resistance of the OBK3 electrode is comparatively low, which would result from the low diffusion resistivity of the electrolyte through the relatively large pores (meso/macropores) of the electrode. In general, a pore widening is caused by a relatively high concentration of activating agent in the presence of many open-pores, through which the activating agent penetrates into the structure. Therefore, pore structure and macroscopic morphology appear to drastically change in the OBK3 sample.

Figure 1 shows the variation of the pore-size distribution (PSD) evaluated by a density functional theory (DFT) method. The OBC sample provided the simple peak of $1.86 \mathrm{~nm}$, accompanied by the large pores of 30 to $200 \mathrm{~nm}$. After $\mathrm{KOH}$ activation, the dominant peak was shifted to the smaller range, providing two peaks at ca. 0.7 and $1.5 \mathrm{~nm}$. The variation manner of the PSD is well consistent with a concept of pore-width development, which results from the repetition of collapse and recombination of pores by $\mathrm{KOH}$ activation. The two dominant peaks (ca. 0.6 and $1.4 \mathrm{~nm}$ ) observed in OBK1 were slightly shifted to the larger values in OBK2, accompanied by the increase in the incremental pore volume. In OBK3, however, the peak at $0.7 \mathrm{~nm}$ has remarkably decreased with the increase in the $1.5 \mathrm{~nm}$-peak and the macropores (> 50nm). This indicates that the small pores grow into the large pores by the coalescence with each other and/or by the collapse of the small pores themselves. In OBK4, the peak at $0.7 \mathrm{~nm}$ increased again with the decrease in the $1.5 \mathrm{~nm}$ peak. It is likely to be due to the re-creation of pores on the morphology stabilized after the collapse over the whole structure. Based on these results, it is concluded that the development of micropores is nearly completed at 2-fold amount of $\mathrm{KOH}$ addition, and further addition of $\mathrm{KOH}$ forms larger size of pores. 
Figure 2 shows the variation of gravimetric $(\mathrm{F} / \mathrm{g})$ and volumetric $(\mathrm{F} / \mathrm{cc})$ capacitances in an aqueous electrolyte system (30 wt. $\% \mathrm{H}_{2} \mathrm{SO}_{4}$ ) as a function of current density and $\mathrm{KOH}$ addition. Two kinds of carbon materials were referred as standards: a poly(vinylidene) chloride (PVDC)-based carbon and a coconut shell-based activated carbon (CSAC) accepted commonly in capacitor industry. The PVDC-based carbon provided the highest capacitance (67 F/g in symmetrically arranged two-electrode system) at the low current density on discharge. However, the rate capability (i.e., ion permeability on a high discharge rate) steeply decreased, compared with the OBK samples. In contrast, the OBK samples are more advantageous in the high discharge current density region ( $>100 \mathrm{~mA} / \mathrm{cm}^{2}$ for $\mathrm{F} / \mathrm{g}$ and $>200 \mathrm{~mA} / \mathrm{cm}^{2}$ for $\mathrm{F} / \mathrm{cc}$ ), compared with the two standards. These bamboo-based ACs can be classified into two groups on the basis of the rate capability; OBK1 and OBK2 (group 1), OBK3 and OBK4 (group 2). The group 2 with a relatively higher mesopore fraction (see Table 1) has shown a higher capacitance at the high discharge current density region $\left(300-800 \mathrm{~mA} / \mathrm{cm}^{2}\right)$. This would result from an effective mobility of electrolyte ions through the large pores (i.e., mesopore). As shown in Fig. 2(c) and (d), the difference of the capacitance observed in the group 2 is relatively small on all current densities. This also demonstrates that the mobility of ions is dependent on the pore width, although the micropores contribute to the charge uptake at a low discharge rate.

To clarify further the correlation between pore and ion sizes for capacitance properties, we carried out the experiments using non-aqueous electrolytes with larger ion radii in an aprotic solvent ( $1 \mathrm{M}$ of $\mathrm{Et}_{4} \mathrm{NBF}_{4}$ in propylene carbonate (PC), ionic radius: $\mathrm{Et}_{4} \mathrm{~N}^{+} ; 0.74$ $\left.\mathrm{nm}, \mathrm{BF}_{4}{ }^{-} ; 0.49 \mathrm{~nm}\right)$. Figure 3 shows the variation of specific capacitance in the organic system for current density and $\mathrm{KOH}$ addition. The OBK3 sample with the highest 
mesopore-to-micropore fraction (see Table 1) provided the maximum specific capacitance (Fig. 3(a), (b)), different from the results in the aqueous system. Furthermore, the OBK3 sample showed the maximum capacitance uptake observed at the low $\left(1 \mathrm{~mA} / \mathrm{cm}^{2}\right)$ and high $\left(40 \mathrm{~mA} / \mathrm{cm}^{2}\right)$ current densities (Fig. 3(c), (d)). The difference of the results in aqueous and organic systems implies that the ion-transfer diffusion with organic solvents will be hindered by narrow pores. In addition, the charge storage has eminently increased between OBK1 and OBK2, and the difference of the capacitance at both current densities becomes larger as the $\mathrm{KOH}$ addition increases (Fig. 3 (c), (d)). This means that the increase from 2- to 3-fold amount of $\mathrm{KOH}$ addition causes a significant change in the pore structure of bamboo-based ACs.

In conclusion, the $\mathrm{KOH}$ activation of bamboo-based char (OBC) at the $\mathrm{OBC} / \mathrm{KOH}$ ratio of 1/3 (OBK3) induces a relatively high mesopore fraction. The OBK3 with the high mesopore fraction showed the maximum capacitance in the organic system and the desirable rate capability in an aqueous electrolyte. The results of this work have demonstrated a potential of bamboo-based ACs for the electrode materials of EDLCs in terms of the better capacitance performance as well as the facility of preparation and the abundance of bamboo-waste materials.

\section{Acknowledgements}

This work was partially supported by the CLUSTER of Ministry of Education, Culture, Sports, Science and Technology of Japan.

\section{References}


[1] Abe I, Fukuhara T, Maruyama J, Tatsumoto H, Iwasaki S. Preparation of carbonaceous adsorbents for removal of chloroform from drinking water. Carbon 2001; 39(7): 1069-73.

[2] Kannan N, Sundaram MM. Kinetics and mechanism of removal of methylene blue by adsorption on various carbons-a comparative study. Dyes and Pigments 2001; 51(1): $25-40$.

[3] Shi H. Activated carbons and double layer capacitance. Electrochim Acta 1996; 41(10): 1633-9.

[4] Laine J, Calafat A, Labady M. Preparation and characterization of activated carbons from coconut shell impregnated with phosphoric acid. Carbon 1989; 27(2): 191-5.

[5] Endo M, Maeda T, Takeda T, Kim YJ, Koshiba K, Hara H, et al. Capacitance and Pore size distribution in aqueous and non-aqueous electrolytes using various activated carbon electrodes. J Electrochem Soc 2001;148(8):A910-4.

[6] Marsh H, Murdie N, Edwards IAS, Boehm H-P. Interactions of Carbons, Cokes, and Graphites with Potassium and Sodium. In: Thrower PA, editor. Chemistry and Physics of Carbon, Vol. 20, New York: Marcel Dekker, 1987:213-72.

[7] Kim YJ, Horie Y, Ozaki S, Matsuzawa Y, Suezaki H, Kim C, et al. Correlation between the pore and solvated ion size on capacitance uptake of PVDC-based Carbons. Carbon 2004; 42 (8-9):1491-500.

\section{The captions for Figures and Table}

Table 1. Fundamental characterizations of chemically activated bamboo char in comparison to bamboo char with only heat treatment at $700{ }^{\circ} \mathrm{C}$ and no chemical activation. 
Figure 1. Pore size distribution (PSD) variation by means of density functional theory (DFT) as a function of $\mathrm{KOH}$ addition. The inset shows a magnification of the pore regime in the macropore-range (30 to $300 \mathrm{~nm}$ ).

Figure 2. Variation of the capacitance obtained using the aqueous electrolyte (30 wt.\% of $\mathrm{H}_{2} \mathrm{SO}_{4}$ ); (a) the $\mathrm{F} / \mathrm{g}$ and (b) the $\mathrm{F} / \mathrm{cc}$ for the discharge current density $\left(\mathrm{mA} / \mathrm{cm}^{2}\right.$ ), and (c) the gravimetric and (d) the volumetric obtained at various current density as a function of the $\mathrm{KOH}$ addition. As a standard, two different carbon candidates were used, one is PVDC based carbon and the other is Coconut shell based AC.

Figure 3. Variation of the specific capacitance in an organic system; (a), (b) were plotted by the current density, and (c), (d) by $\mathrm{KOH} /$ char ratio. The difference of the capacitance depend on the current density has also been illustrated. 
- Table 1. -

\begin{tabular}{|c|c|c|c|c|c|c|c|c|c|c|c|}
\hline Samples & $\begin{array}{l}\text { Yield }^{a} \\
\text { (wt.\%) }\end{array}$ & $\begin{array}{c}\mathrm{S}_{\mathrm{BET}} \\
\left(\mathrm{m}^{2} / \mathrm{g}\right)\end{array}$ & $\begin{array}{l}S_{\text {micro }} \\
\left(\mathrm{m}^{2} / \mathrm{g}\right)\end{array}$ & $\mathrm{S}_{\text {meso }} / \mathrm{S}_{\text {micro }}$ & $\begin{array}{c}\mathrm{V}_{\text {Tot }} \\
\left(\mathrm{cm}^{3} / \mathrm{g}\right)\end{array}$ & $\begin{array}{c}\mathrm{V}_{\text {micro }} \\
\left(\mathrm{cm}^{3} / \mathrm{g}\right)\end{array}$ & $\mathrm{V}_{\text {meso }} / \mathrm{V}_{\text {micro }}$ & $\begin{array}{l}w^{b} \\
(\mathrm{~nm})\end{array}$ & $\begin{array}{l}\text { ESR } \\
(\Omega)\end{array}$ & $\begin{array}{c}\rho^{c} \\
\left(\mathrm{~g} / \mathrm{cm}^{3}\right)\end{array}$ & $\begin{array}{c}\omega^{d} \\
(\Omega \square \mathrm{m})\end{array}$ \\
\hline $\mathrm{OBC}^{e}$ & 30 & 246.77 & 225.47 & 0.09 & 0.123 & 0.108 & 0.14 & 4.33 & 1.149 & 0.95 & $3.57 \times 10^{-2}$ \\
\hline $\mathrm{OBK}^{f}$ & 75 & 1010.21 & 913.41 & 0.11 & 0.489 & 0.434 & 0.13 & 2.56 & 0.142 & 0.77 & $4.25 \times 10^{-4}$ \\
\hline OBK2 & 69 & 1251.67 & 1027.24 & 0.22 & 0.609 & 0.493 & 0.24 & 2.62 & 0.171 & 0.68 & $4.45 \times 10^{-4}$ \\
\hline OBK3 & 60 & 1293.20 & 894.64 & 0.45 & 0.634 & 0.429 & 0.48 & 2.86 & 0.134 & 0.60 & $5.48 \times 10^{-4}$ \\
\hline OBK4 & 51 & 1413.21 & 1034.27 & 0.37 & 0.708 & 0.506 & 0.40 & 3.01 & 0.146 & 0.63 & $7.22 \times 10^{-4}$ \\
\hline
\end{tabular}

${ }^{a}$ The yields of the OBC was obtained from the weight of the as-received bamboo and the OBKs' were calculated from a weight of the OBC.

${ }^{b}$ Average pore width in diameter

${ }^{c}$ Apparent density of electrode including a binder (5 wt.\% of PTFE)

${ }^{d}$ Powder resistance obtained from the pressure of 34.3 MPa

${ }^{e}$ bamboo char from the $\mathrm{HTT}=700^{\circ} \mathrm{C}$ for $2 \mathrm{~h}$

${ }^{f}$ activated at bamboo char/KOH ratios $=1: 1(\mathrm{OBK} 1) \sim 1: 4(\mathrm{OBK} 4)$ 


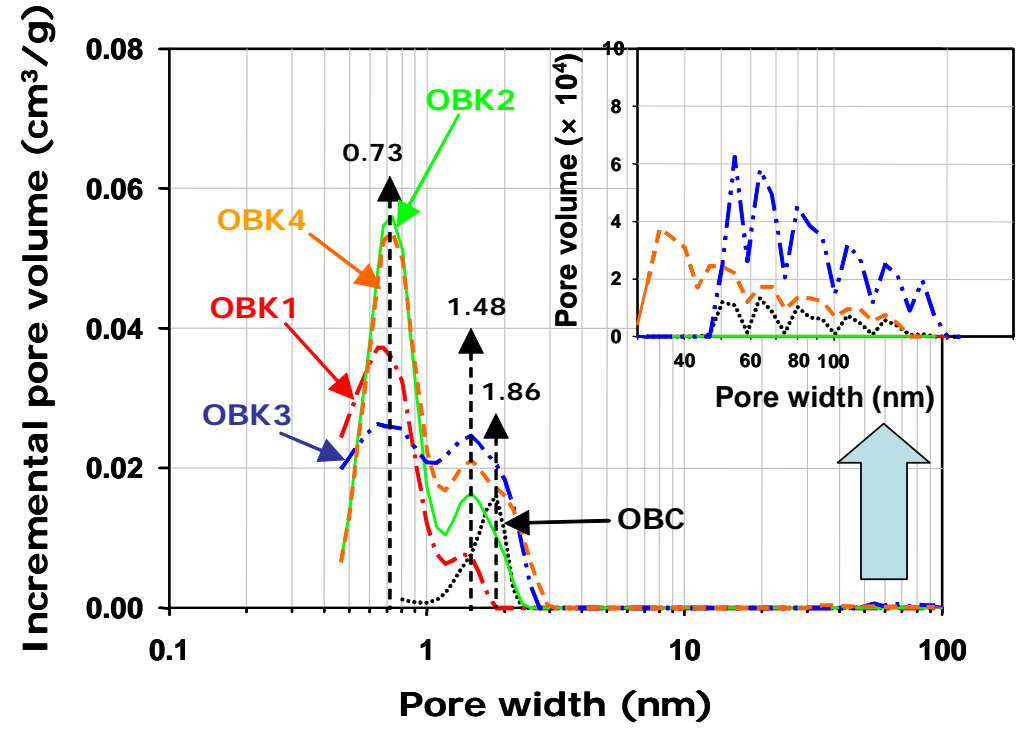

- Fig. 1 - 

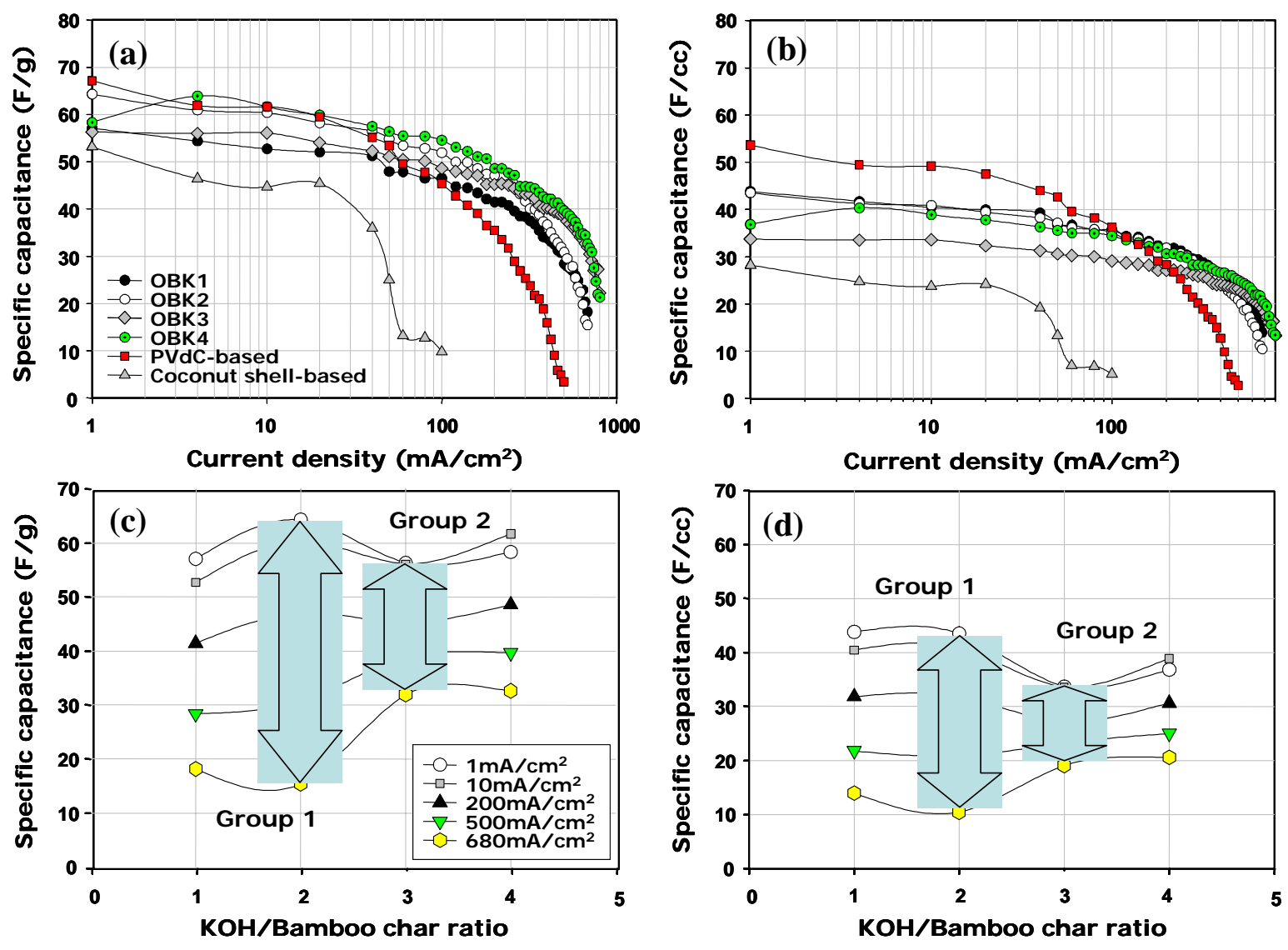

- Fig. 2 - 

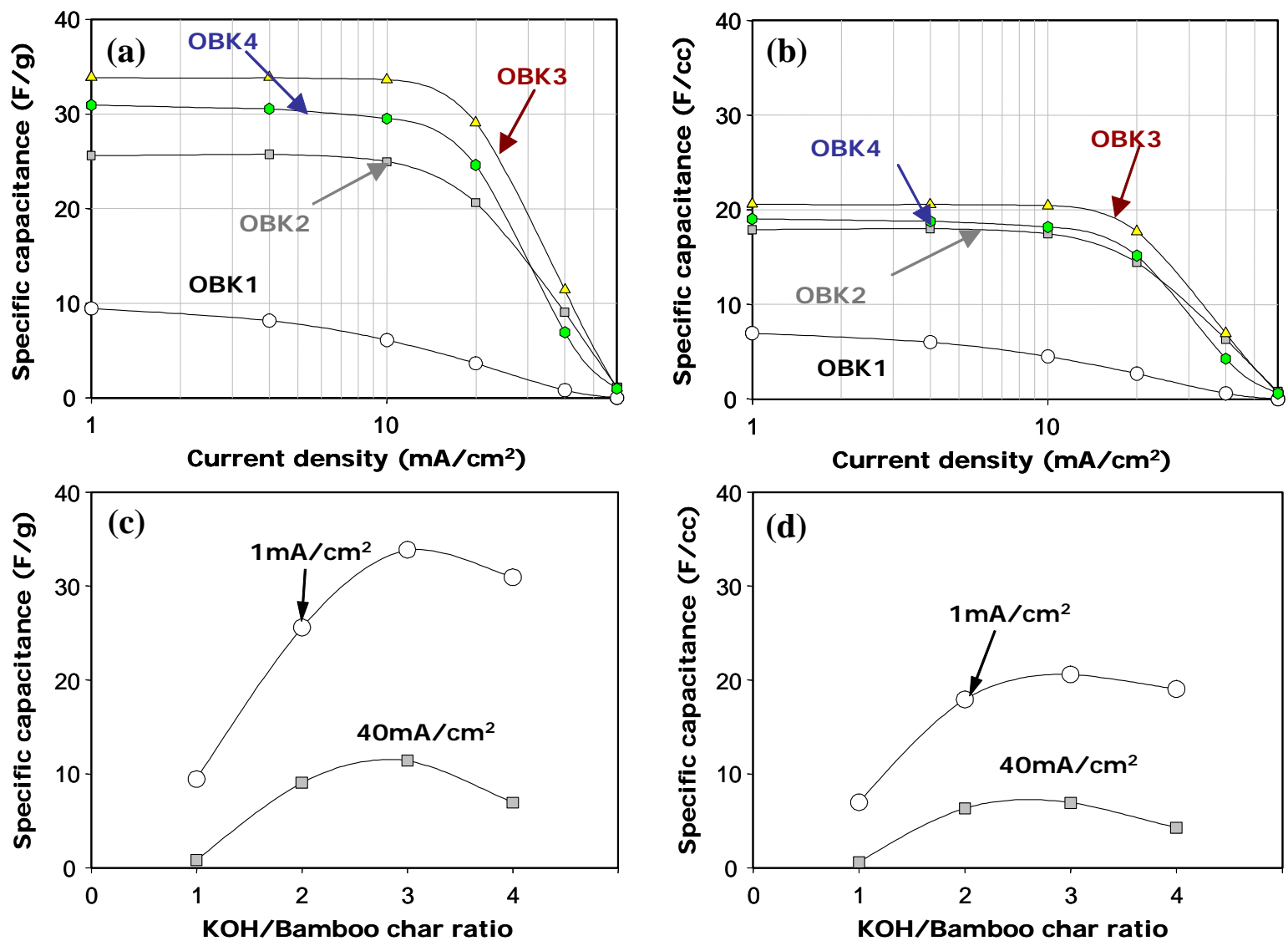

- Fig. 3 - 